\title{
5G Contender Waveforms for Low Power Wide Area Networks in a 4G OFDM Framework
}

\author{
Yoann RoTH ${ }^{1,2}$, Jean-Baptiste DoRÉ ${ }^{2}$, Laurent Ros ${ }^{1}$ and Vincent BERG ${ }^{2}$ \\ ${ }^{1}$ Univ. Grenoble Alpes, CNRS, Grenoble INP*, GIPSA-lab, 38000 Grenoble, France \\ ${ }^{2}$ CEA, LETI, MINATEC Campus, F-38054 Grenoble, France \\ * Institute of Engineering Univ. Grenoble Alpes \\ \{yoann.roth, laurent.ros\}@gipsa-lab.fr, \{jean-baptiste.dore, vincent.berg\}@cea.fr
}

\begin{abstract}
When designing new solutions for Low Power Wide Area (LPWA) networks, coexistence and integration into existing 4G frameworks should be considered to ease the deployment procedure and reduce costs. In a previous work, Turbo-Frequency Shift Keying (FSK) was proposed as a potential physical layer for LPWA networks. We propose to study the system in the Orthogonal Frequency Division Multiplexing (OFDM) framework, currently used by existing cellular networks and also considered for the recently standardized Narrow-Band IoT. The new architectures of the Turbo-FSK transmitter and receiver are presented. Simulations are run under various conditions including Rayleigh fading channel with mobility, demonstrating the Turbo-FSK scheme to achieve performance close to TurboCoded OFDM. The scheme also exhibits a constant envelope, which may multiply the Power Amplifier (PA) efficiency by up to a factor of 2 and makes the solution a promising candidate for 5G LPWA transmission.

Index Terms-LPWA, Turbo Codes, Constant Envelope, OFDM, FSK.
\end{abstract}

\section{INTRODUCTION}

The next generation of cellular Internet-of-Things (IoT) is in the process of being standardized [1] and is expected to interconnect things using existing communication technologies but also evolving technologies [2]. It is beneficial to design new technologies that can be integrated in existing frameworks, e.g the Orthogonal Frequency Division Multiplexing (OFDM) framework used in Long Term Evolution (LTE) systems (4G). The framework defines the structure of the signal and the general parameters of the physical waveforms. By considering an existing framework, deployment costs are reduced and coexistence of various technologies is facilitated.

In the IoT and 5G context, Low Power Wide Area (LPWA) networks [3], [4] are expected to represent approximately $10 \%$ of the overall IoT connections [5]. Requirements for LPWA include long range communication and low energy consumption at the device level, hence long battery life. Long range can be achieved by ensuring a very low level of sensitivity at the receiver. This can be achieved by allowing the Quality of Service (QoS) to be reached for low levels of Signal-to-Noise Ratio (SNR) [6].

Narrow-Band IoT (NB-IoT), introduced in the latest LTE release [7]-[10], relies on this strategy. The uplink transmission of this solution combines the powerful (13 15) 8 Turbo Code (TC) [7], [11] as Forward Error Correction (FEC) and a repetition factor guarantees QoS at low levels of SNR. DFT-spread OFDM as well as single tone waveforms are part of the standard. It is highly energy efficient and offers a flexibility in data rate that makes it able to match numerous applications. Another solution relying on the same strategy is Turbo-Frequency Shift Keying (Turbo-FSK) [6].
Low levels of sensitivity are achieved thanks to the use of a combination of orthogonal modulation, a convolutional code and an iterative receiver. The scheme also offers a constant envelope modulation which reduces the constraints imposed on the Power Amplifier (PA). While Turbo-FSK has solely been presented as an alternative to other standalone solutions for LPWA, the use of Frequency Shift Keying (FSK) modulation allows to integrate the technology in an OFDM framework. Indeed, both FSK and OFDM modulations can be generated and demodulated using the Fast Fourier Transform (FFT) algorithm. More than a standalone technology, Turbo-FSK can thus be used as an additional mode for LPWA communications in the LTE context. On one hand the single tone scheme of the NB-IoT cannot fullfill some latency requirements for certain IoT applications. On the other hand, the DFT-spread strategy allows higher throughput and lower latency at the price of a reduction of the power amplifier efficiency due to higher envelope fluctuation. The proposed scheme allows to increase throughput and reduced latency while maintaining constant envelope signal property.

In this paper, we propose an extensive study of TurboFSK when included in an OFDM framework. The scheme is compared to other solutions based on the $(1315)_{8}$ TC, namely Turbo Coded Orthogonal Frequency Division Multiplexing (TC-OFDM), Turbo Coded Single Carrier Frequency Division Multiple Access (TC-SC-FDMA) and Turbo Coded Frequency Shift Keying (TC-FSK). The first two schemes are directly inspired by the NB-IoT solution, while the third one enables a comparison of Turbo-FSK with another turbo coded constant envelope waveform. After the presentation of the four schemes in the OFDM framework, performance is compared. Four aspects are studied: performance under Additive White Gaussian Noise (AWGN), performance under static Rayleigh fading channel, performance under Rayleigh fading channel with mobility and variations of the envelope. A low rate scenario is considered for the simulations.

The paper is organized as follows. The system model, including the OFDM framework and the four compared solutions, is presented in Section II. Performance is compared in Section III and Section IV concludes the paper.

\section{SYSTEM MODEL}

This section is dedicated to the presentation of the general OFDM framework and of the various techniques that are evaluated and compared. 


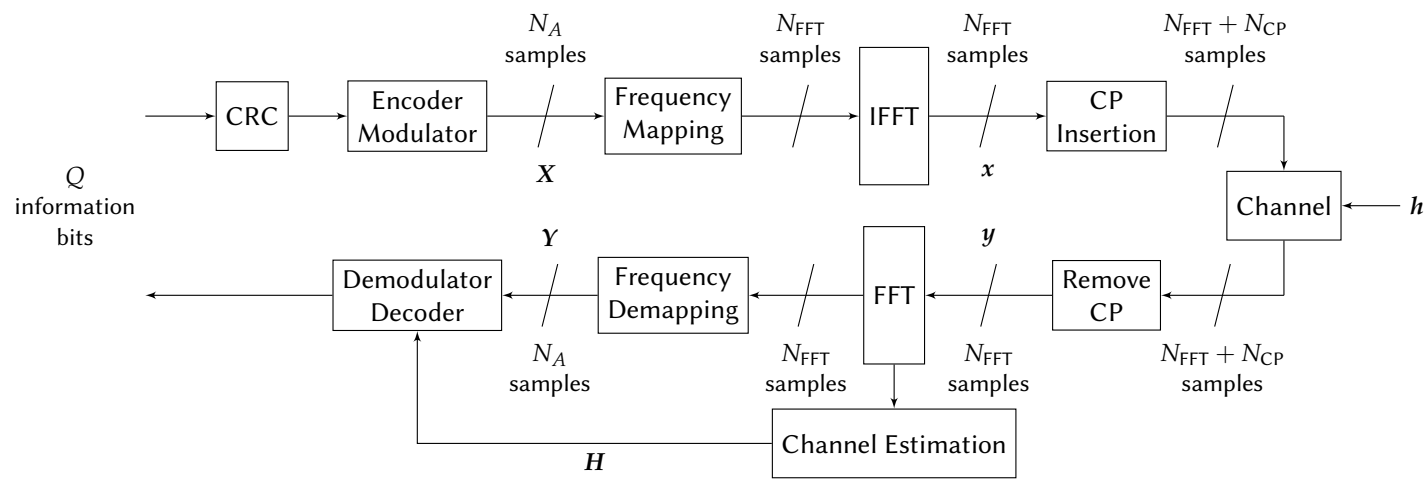

Figure 1: System model for the OFDM framework.

\section{A. OFDM Framework}

The general principle of the OFDM framework is to construct the signal in the frequency domain. Over the $N_{\text {FFT }}$ available carrier frequencies, only $N_{A}$ are considered to transmit information. This is a form of multicarrier modulation. The sequence generation in the OFDM framework, i.e. the transmitter architecture, and the considered channel are described first. Then, assuming a generic form for the transmitted sequence, the mathematical derivations of the likelihood of a sequence are computed. The result will be used in further sections to derive the probabilistic receiver of the considered schemes. The generic architecture of the OFDM framework is finally presented, along with the values of the parameters considered for the simulations.

1) Transmitter: The OFDM framework is depicted in Figure 1. From the information packet of size $Q$, a Cyclic Redundancy Check (CRC) is computed and appended to the information block. Encoding and modulation steps are then performed. These steps depend on the choice of the technique and are presented later. The output of this block is represented by a matrix of size $N_{A} \times N_{s}$, where $N_{A}$ is the number of active frequencies and $N_{s}$ the number of time slots (or OFDM symbols). This is the time-frequency allocation which is then included in the FFT grid of size $N_{\mathrm{FFT}} \times N_{s}$. Inverse Discrete Fourier Transform (IDFT) of size $N_{\mathrm{FFT}}$ is then applied to convert the frequency signal into a time signal consisting of $N_{\text {FFT }} \times N_{s}$ samples (or chips). The Inverse Fast Fourier Transform (IFFT) algorithm is used in practice to perform the IDFT. Considering the vector $\boldsymbol{X}$ of length $N_{\mathrm{FFT}}$, the considered OFDM symbol $\boldsymbol{x}$ is given by

$$
x(k)=\frac{1}{N_{\mathrm{FFT}}} \sum_{n=0}^{N_{\mathrm{FFT}}-1} X(n) e^{j 2 \pi \frac{k n}{N_{\mathrm{FFT}}}}
$$

which is the IDFT of the vector $\boldsymbol{X}$. A Cyclic Prefix (CP) of length $N_{\mathrm{CP}}$ consisting of the last $N_{\mathrm{CP}}$ samples of each OFDM symbol is then inserted at the beginning of each symbol.

2) Channel: The emitted signal then transits through the channel. The use of a $\mathrm{CP}$ allows the effect of the channel to be expressed by a circular convolution when the channel delay spread is smaller than the size of the CP. Multi-path channel is modelled by a discrete-time domain representation i.e. a Finite
Impulse Response (FIR) filter applied at chip-time. The useful signal (after removing the $\mathrm{CP}$ ) at index $k$ is given by

$$
y(k)=\sum_{l=0}^{L_{\mathrm{ch}}-1} h(l) x(k-l)+\nu(k),
$$

where $\boldsymbol{h}$ is the channel impulse response, a complex vector with $L_{\mathrm{ch}}$ elements, representing the gain of each possible paths. The elements $\nu_{k}$ of the noise vector $\nu$ are independent and follow a circularly-symmetric and zero mean complex normal distribution with variance $\sigma_{\mathrm{ch}}^{2}$. The circular convolution assumption can only be considered if $N_{\mathrm{CP}} \geq L_{\mathrm{ch}}$.

3) Likelihood Computation: In order to derive the probabilistic receiver for each technique, we would like to express the likelihood of observing a sequence $\boldsymbol{y}$ given that a sequence $\boldsymbol{x}$ was transmitted. A generic form for the transmitted sequence is considered, and further simplifications will occur when specific modulation techniques are considered. The likelihood of observing a sequence $\boldsymbol{y}$ given that a sequence $\boldsymbol{x}$ was transmitted is given by

$$
p(\boldsymbol{y} \mid \boldsymbol{x})=\prod_{k=0}^{N_{\mathrm{FFT}}-1} p(y(k) \mid x(k)) .
$$

As normal distribution with variance $\sigma_{\mathrm{ch}}^{2}$ was assumed, the likelihood is expressed

$$
\begin{aligned}
p(\boldsymbol{y} \mid \boldsymbol{x}) & =C_{\mathrm{st}} \exp \left\{-\frac{1}{2 \sigma_{\mathrm{ch}}^{2}} \sum_{k=0}^{N_{\mathrm{FFT}}-1}\left|\sum_{l=0}^{L_{\mathrm{ch}}-1} h(l) x(k-l)\right|^{2}\right. \\
& \left.+\frac{1}{\sigma_{\mathrm{ch}}^{2}} \sum_{k=0}^{N_{\mathrm{FFT}}-1}\left\langle y(k), \sum_{l=0}^{L_{\mathrm{ch}}-1} h(l) x(k-l)\right\rangle\right\},
\end{aligned}
$$

where $C_{\text {st }}$ is a term independent of $\boldsymbol{x}$ and $\langle\cdot, \cdot\rangle$ is the scalar product operator.

Using (1) in (2), the likelihood can be expressed with (3), where $Y(n)$ (resp. $H(n))$ is the $n$-th element of the Discrete Fourier Transform (DFT) of size $N_{\text {FFT }}$ of the vector $\boldsymbol{y}$ (resp. $\boldsymbol{h}$ ). As the form of $\boldsymbol{x}$ was purposely kept general, additional computations are required to obtained the expression of the Maximum Likelihood (ML) decoder. However, the expression demonstrates the necessity for the receiver to evaluate the DFT of the received time-vector $\boldsymbol{y}$ denoted by $\boldsymbol{Y}$, and to have knowledge of $\boldsymbol{H}$, the DFT of the channel coefficients. 


$$
p(\boldsymbol{y} \mid \boldsymbol{x})=C_{\mathrm{st}} \exp \left\{-\frac{1}{2 \sigma_{\mathrm{ch}}^{2}} \frac{1}{N_{\mathrm{FFT}}^{2}} \sum_{k=0}^{N_{\mathrm{FFT}}-1}\left|\sum_{n=0}^{N_{\mathrm{FFT}}-1} X(n) H(n) e^{j 2 \pi \frac{n k}{N_{\mathrm{FFT}}}}\right|^{2}+\frac{1}{\sigma_{\mathrm{ch}}^{2}} \frac{1}{N_{\mathrm{FFT}}} \operatorname{Re}\left(\sum_{n=0}^{N_{\mathrm{FFT}}-1} \overline{X(n) H(n)} Y(n)\right)\right\}
$$

The Log Likelihood Ratio (LLR) of the bit $b_{m}$ is given by

$$
L\left(b_{m}\right)=\log \frac{p\left(\boldsymbol{y} \mid b_{m}=+1\right)}{p\left(\boldsymbol{y} \mid b_{m}=-1\right)}=\log \frac{\sum_{i \in \mathcal{X}_{+1}^{m}} p\left(\boldsymbol{y} \mid \boldsymbol{x}^{i}\right)}{\sum_{i \in \mathcal{X}_{-1}^{m}} p\left(\boldsymbol{y} \mid \boldsymbol{x}^{i}\right)},
$$

where $\mathcal{X}_{+1}^{m}$ (resp. $\mathcal{X}_{-1}^{m}$ ) the group of sequences $\boldsymbol{x}$ which encode an information word for which the bit $b_{m}$ is equal to +1 (resp. -1 ). The LLR of the bits for each technique will be computed using the specific expression of the sequence $\boldsymbol{x}$ in (3) and (4).

4) Receiver: In order to retrieve the information that was mapped in the frequency domain at the transmitter side, a DFT of size $N_{\text {FFT }}$ is applied on each OFDM symbol, using the FFT algorithm. The result is the vector $\boldsymbol{Y}$, the channel observation. By selecting only the $N_{A}$ used frequencies, the $N_{s}$ vectors of size $N_{A}$ transmitted at the first place are retrieved. This signal is fed to the demodulator/decoder along with $\boldsymbol{H}$.This vector can also be interpreted as a complex gain on each frequency. Using the channel observation and the vector $\boldsymbol{H}$, the block demodulator uses a simplified formulation of (3) to compute the LLR of the transmitted bits. The information bits are finally estimated by the decoder and the CRC is computed to evaluate if the packet contains any error.

\section{B. TC-OFDM and TC-SC-FDMA}

Two well known techniques are considered for comparison: the use of OFDM associated with the $(1315)_{8}$ TC [12] and the TC-SC-FDMA, for which a pre-coding based on a DFT transform is applied. These schemes are multiplexing techniques, i.e. a Quadrature Phase Shift Keying (QPSK) symbol is mapped on each frequency carrier. For both techniques, the Encoder/Modulator block of Figure 1 uses a TC encoder and a QPSK modulator. However, since there are $N_{A} N_{s}$ available symbols, a rate matching mechanism is used to repeat or puncture some bits. The rate $R_{m}$ corresponds to the ratio between the number of input bits $(3 Q+12)$ of the rate matcher and the number of output bits $\left(2 N_{A} N_{s}\right)$. If $R_{m}>1$, then bits are punctured (with interleaving to ensure that the punctured bits are evenly spread over time), and when $R_{m}<1$, bits are repeated. An interleaver is used between the encoding and modulation processes. For the case of TC-SC-FDMA, precoding using a DFT of size $N_{\text {DFT }}$ is applied.

While the derivations of the receivers could be obtained using the general expression of the likelihood (3), these computations are well documented in the literature [13] and are not detailed here. For the TC-SC-FDMA technique, the ML receiver is too complex to be considered. The Zero-Forcing (ZF) equalizer is used instead. For the turbo decoder, the max$\log$ approximation is considered and the CRC is used as stop criterion for the iterations of the decoder, with a maximum number of iterations set to 10 .

\section{Turbo-FSK}

The Turbo-FSK principle was introduced in [6]. The motivation for this scheme was to operate close to the Shannon capacity [14] while using a constant envelope modulation. Turbo-FSK can be implemented using an OFDM transceiver. The technique relies on the use of the FSK modulation associated with a convolutional code and an iterative decoder. FSK is a common orthogonal modulation, based on an alphabet of orthogonal waveforms equivalent to pure frequencies. When using the previously introduced OFDM framework, only one of the $N_{A}$ possible carriers is actived every time slot. The information is carried by the index of the activated carrier. The innovative aspect of the Turbo-FSK scheme is in the association of the encoding and modulation processes at the transmitter side. The turbo receiver exploits this association to operate at very low levels of SNR.

The Encoder/Modulator block for the Turbo-FSK is depicted in Figure 2. Similarly to the transmitter presented in [6], the encoder consists in $\lambda$ stages where each stage encodes an interleaved version of the $Q$ information bits. The information block is divided into $N_{q}$ information words of $q$ bits. Eventually, a total of $\lambda \times\left(N_{q}+1\right)$ binary words of length $q+1$ are generated. Each binary word is mapped on one of the codewords of the alphabet. In order to fit the $N_{s}$ available symbols, the rate matcher punctures or repeats some binary words so that

$$
R_{m}=\frac{\lambda \cdot\left(N_{q}+1\right)}{N_{s}} .
$$

Unlike the other schemes, the rate matcher operates at the word level instead of operating at the bit level. This is because entire words of size $q+1$ are associated to one of the symbol of the selected alphabet. It is composed of $M=2^{q+1}$ pure frequencies. If the binary word $\boldsymbol{b}$ is associated to the frequency $i$, then the output of the modulator is

$$
X(n)=\left\{\begin{array}{ll}
1 & \text { if } n=i \\
0 & \text { otherwise }
\end{array} .\right.
$$

The Demodulator/Decoder block is depicted in Figure 2. The first operation is to compute the likelihood of all the symbols from the alphabet. When combining the general expression (3) with (5), the likelihood given that the frequency $i$ was sent is expressed

$$
\begin{aligned}
p\left(\boldsymbol{y} \mid \boldsymbol{x}^{i}\right)=C_{\mathrm{st}} \exp \left\{\frac{1}{\sigma_{\mathrm{ch}}^{2} N_{\mathrm{FFT}}}(\right. & \operatorname{Re}(\overline{H(i)} Y(i)) \\
& \left.\left.-\frac{1}{2}|H(i)|^{2}\right)\right\} .
\end{aligned}
$$

After computing the likelihood of each sequence, a matrix of size $M \times N_{s}$ is obtained, containing the likelihoods of all the possible symbols from the alphabet. The puncturing management block is then in charge to recover the original 


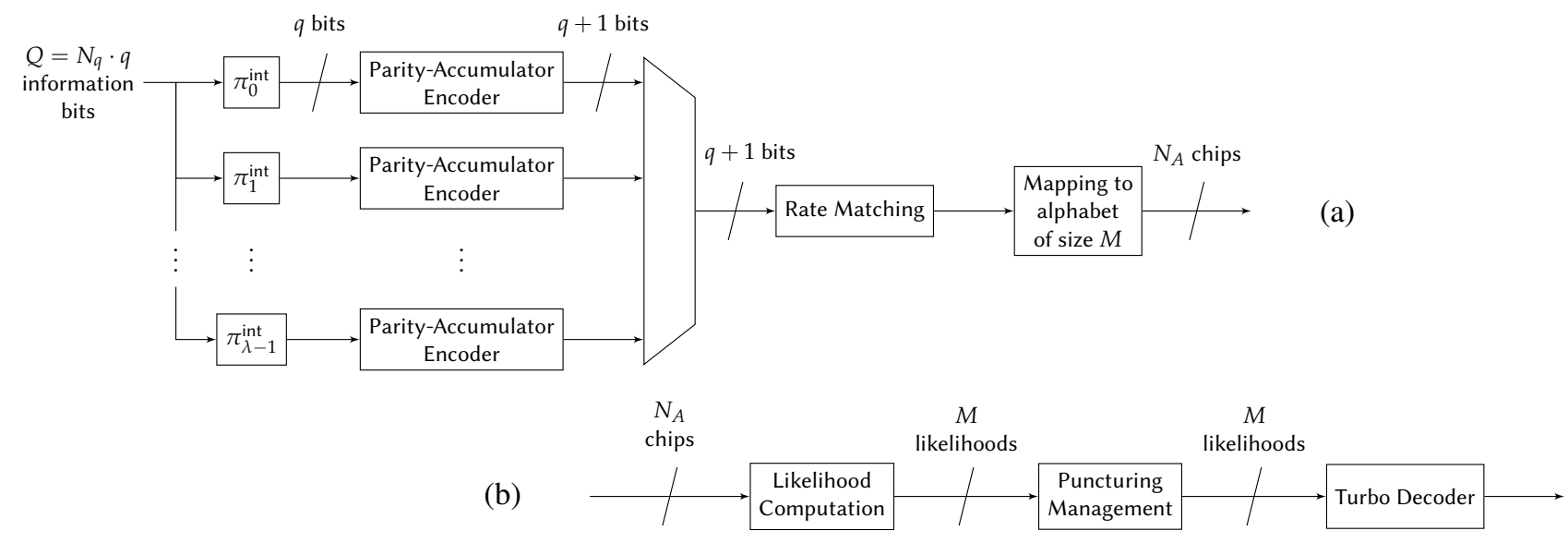

Figure 2: (a) Encoder/Modulator and (b) Demodulation/Decoder for the Turbo-FSK system.

$M \times \lambda\left(N_{q}+1\right)$ matrix generated at the transmitter. If a symbol was repeated, columns corresponding to the repetitions of this symbol are added. If a symbol was punctured, a column of $0 \mathrm{~s}$ is added. The resulting matrix corresponds to the channel observation of all stages. The turbo decoder then uses the observation of the $\lambda$ stages to perform the iterative decoding presented in [6] (detailed in [15]). The CRC can be used as a stop criteria, as it is computed at the end of each iteration.

\section{TC-FSK}

The use of FSK modulation associated with the $\left(\begin{array}{lll}13 & 15\end{array}\right)_{8}$ TC can also be considered. For this scheme, the transmitter performs turbo encoding associated with a rate matching mechanism and an interleaver. FSK modulation is applied and the output is expressed with (5). The receiver computes the LLR of the binary sequence using

$$
\begin{aligned}
L\left(b_{m}\right) & =\max _{i \in \mathcal{X}_{1}^{m}}\left[\operatorname{Re}(\overline{H(i)} Y(i))-\frac{1}{2}|H(i)|^{2}\right] \\
& -\max _{i \in \mathcal{X}_{-1}^{m}}\left[\operatorname{Re}(\overline{H(i)} Y(i))-\frac{1}{2}|H(i)|^{2}\right],
\end{aligned}
$$

which is obtained by combining Equation (6), Equation (4) and the max-log approximation. Turbo decoding is then performed, similarly to the TC-OFDM.

\section{Performance}

In order to compare the performance of the various schemes, we select specific values for the parameters of the OFDM framework. The parameters are given in Table I, where $f_{s}$ is the sampling frequency, $\Delta f$ the subcarrier spacing and $f_{c}$ the carrier frequency. The spectral efficiency can be computed with

$$
\eta=\frac{Q}{N_{s}\left(N_{\mathrm{FFT}}+N_{\mathrm{CP}}\right)}
$$

and the data rate is given by $R=Q / N_{s}$. With the selected parameters, the data rate is equal to $R=8.24 \mathrm{kbps}$. This rather low value is consistent with the fact that long range solutions must transmit at low data rates to provide low sensitivity levels [6]. Since the number of symbols is set to $N_{s}=1700$, the rate matcher of each technique has a different output rate. The

\begin{tabular}{ccccccc}
$N_{A}$ & $N_{s}$ & $N_{\mathrm{FFT}}$ & $N_{\mathrm{CP}}$ & $f_{s}$ & $\Delta f$ & $f_{c}$ \\
\hline 16 & 1700 & 128 & 9 & $1.92 \mathrm{MHz}$ & $15 \mathrm{kHz}$ & $2.5 \mathrm{GHz}$
\end{tabular}

Table I: Parameters for the OFDM framework. The spectral efficiency is equal to $\eta=4.29 \cdot 10^{-3} \mathrm{bits} / \mathrm{s} / \mathrm{Hz}$.

\begin{tabular}{c|c|c|c|c} 
Scheme & TC-OFDM & TC-SC-FDMA & TC-FSK & Turbo-FSK \\
$R_{m}$ & 0.0563 & 0.0563 & 0.45 & 1 \\
$1 / R_{m}$ & 17.76 & 17.76 & 2.22 & 1
\end{tabular}

Table II: Value of the rate matching for the various techniques selected.

rates of the different schemes are given in Table II, along with the equivalent number of repetitions given by $1 / R_{m}$.

For this comparison, we select the specific value $\lambda=5$ for Turbo-FSK. According to the results found in [16], it is the value of $\lambda$ that gives the lowest $E_{b} / N_{0}$ for this size of alphabet (16-FSK).

For all simulations, perfect synchronization is assumed, along with ideal channel estimation. The iterative decoders perform a maximum of 10 iterations.

\section{A. Under AWGN}

The AWGN channel, i.e. a channel with a single and constant propagation path, is first considered. The Packet Error Rate (PER) performance of the 4 different techniques is depicted in Figure 3, versus the SNR. Very low levels of SNR are considered. The TC-OFDM and TC-SC-FDMA schemes are equivalent under AWGN, and offer the best performance. Turbo-FSK only exhibits a $0.2 \mathrm{~dB}$ loss at a PER of $10^{-3}$. Better performance could be achieved by selecting other combinations of alphabet size and number of repetition $\lambda$ [16]. The TC-FSK suffers from a loss of $2.9 \mathrm{~dB}$. Unlike Turbo-FSK, the receiver of this scheme does not combine FSK demodulation and turbo decoding in the same procedure. Better performance is achieved using a more sophisticated receiver such as the one considered in Turbo-FSK.

\section{B. Under Static Rayleigh Fading Channel}

Simulations under static Rayleigh fading channel are considered. The Extended Typical Urban (ETU) profile [17, p. 


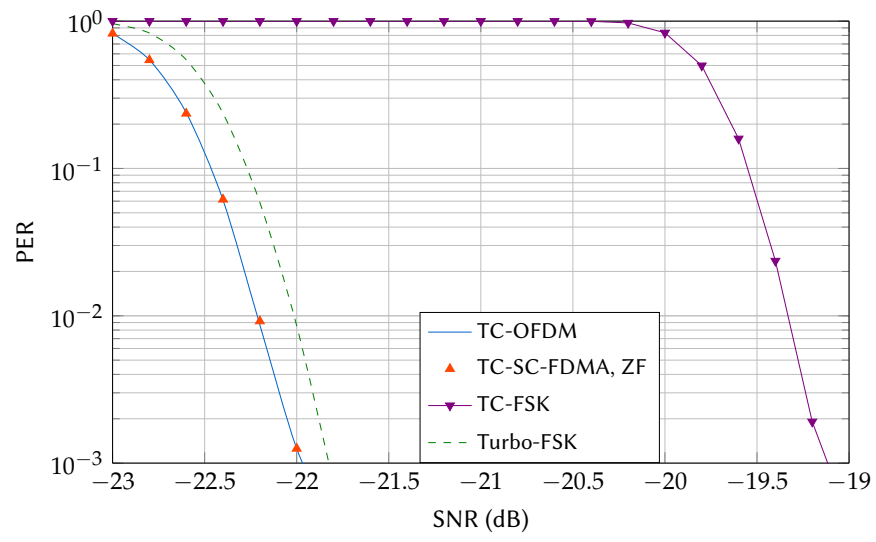

Figure 3: Performance of the four compared solutions under AWGN.

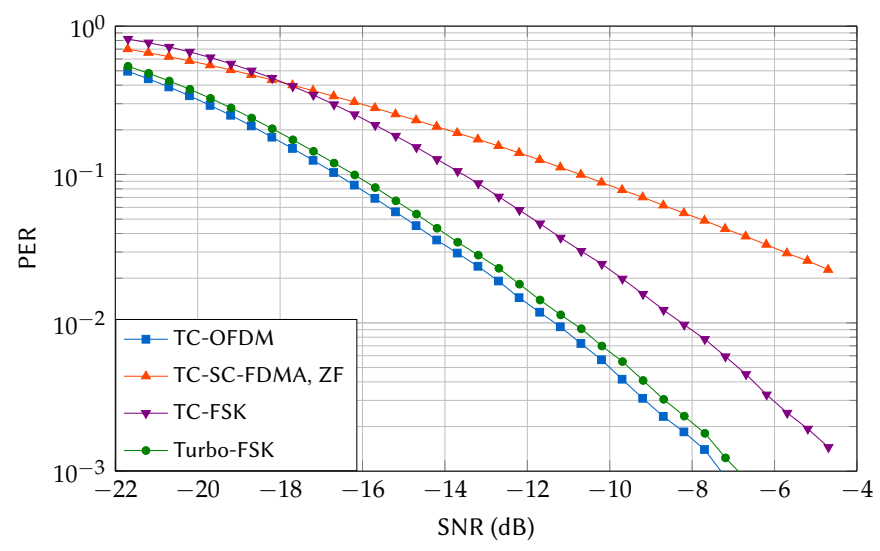

Figure 4: Performance of the four compared solutions under static Rayleigh fading channel with ETU profile.

191], a channel exhibiting severe multipath fading propagation conditions, is considered for the path gains and delays. Static channel condition is evaluated, i.e. there is no relative velocity between the transmitter and the receiver. The performance of the four schemes is depicted in Figure 4. TC-OFDM outperforms all the other schemes with a PER of $10^{-2}$ at $-11.4 \mathrm{~dB}$ of SNR. Performance of Turbo-FSK is only $0.2 \mathrm{~dB}$ away from TC-OFDM. TC-FSK exhibits a loss of $3.1 \mathrm{~dB}$ versus TC-OFDM, as in the AWGN case. The performance loss compared to Turbo-FSK can be incurred to the use of a different decoder, as the signaling technique is identical. TC-SC-FDMA exhibits the worst performance, but this can be incurred to the choice of the ZF equalizer. Other equalizer could be considered such as the Minimum Mean Square Error (MMSE) and may lead to better performance.

\section{Under Rayleigh Fading Channel with Mobility}

A situation of mobility is now considered. The ETU fading profile is considered. The relative velocity between the transmitter and the receiver is equal to $v=50 \mathrm{~km} / \mathrm{h}$. With the selected carrier frequency, given in Table I, the Doppler frequency is equal to $f_{d}=115.8 \mathrm{~Hz}$, giving a coherence time of $T_{\text {coh }}=8.63 \mathrm{~ms}$. The coherence is thus 129 times greater than the symbol time (equal to $66.6 \mu \mathrm{s}$ ). The receiver can assume

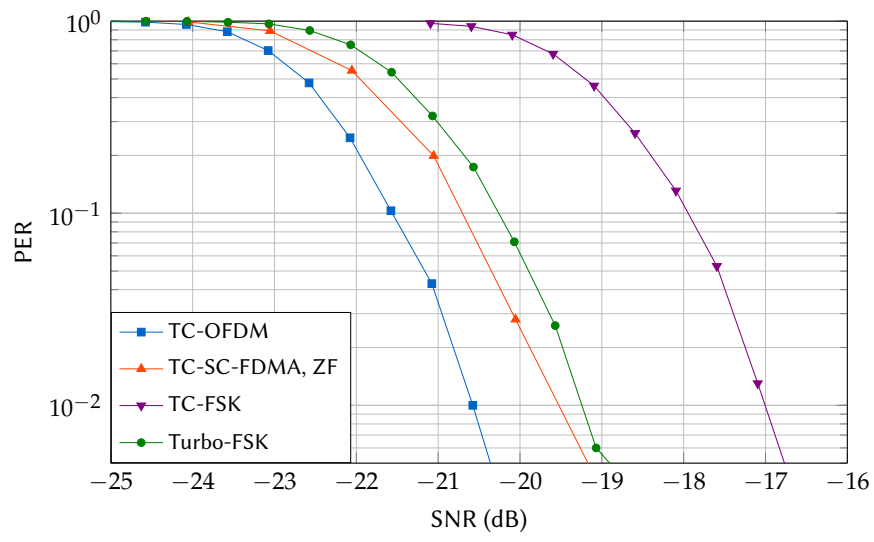

Figure 5: Performance of the four compared solutions under Rayleigh fading channel with ETU profile. The relative speed is equal to $50 \mathrm{~km} \cdot \mathrm{h}^{-1}$ and the carrier frequency is set to $2.5 \mathrm{GHz}$.

a constant vector $\boldsymbol{H}$ for each symbol, which is actually the time-average estimation of the true channel.

The performance of the four schemes under mobility condition is depicted in Figure 5. When compared to the case of the static ETU depicted in Figure 4, mobility significantly improves the performance of all the schemes. The schemes benefit from a large redundancy (for example, the encoded bits for TC-OFDM and TC-SC-FDMA are repeated more than 17 times, and the TC itself offers redundancy). In the mobility case, the same bit (or symbols) and its repetitions experience different channels gains. Far enough appart repetitions experience gains are partially uncorellated, and the fading effect of the channel is averaged over time. This is a form of time diversity and performance tends toward the AWGN case [18]. While the TC-SC-FDMA exhibited poor performance in the static ETU case, the time diversity offered by the repetitions overcomes the negative impact of equalization and the scheme is less than $1 \mathrm{~dB}$ away from TC-OFDM. The TC-FSK scheme is $3.5 \mathrm{~dB}$ away from the TC-OFDM technique. The difference of performance in the static ETU case was in the same order of magnitude. Thanks to the use of repetition and a low spectral efficiency modulation technique, the TC-FSK scheme also benefits from diversity. Turbo-FSK now exhibits a $0.6 \mathrm{~dB}$ gap versus TC-OFDM. It uses $\lambda=5$ repetitions, which offers a diversity comparable to the other techniques.

\section{IAPR}

The variations of the envelope of a signal can be measured through the computation of the Instantaneous-to-Average Power Ratio (IAPR). This measure is very useful for evaluating the constraints of linearity imposed on the PA. It is more relevant than the measure of the Peak to Average Power Ratio (PAPR) as it considers all the samples of the signal (and potentially, the samples that could reach the non-linear region of the PA) [19] instead of only the maximum value. Having large variations will result in the necessity of having a highly linear PA, i.e. expensive and energy inefficient. The IAPR is measured from the time signal which is sent through the channel, after the addition of the $\mathrm{CP}$ in Figure 1. For 


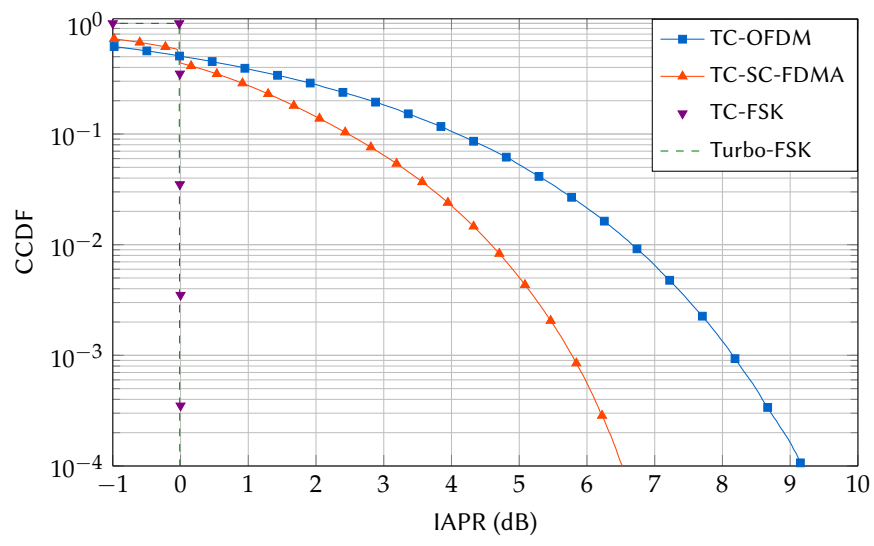

Figure 6: IAPR of the four compared solutions.

a sufficient statistic, 1000 packets are simulated using the parameters of Table I.

The Complementary Cumulative Distribution Function (CCDF) of the IAPR is depicted for the four schemes in Figure 6. TC-OFDM has the largest variations and exhibits a probability of $10^{-3}$ to have an IAPR larger than $8 \mathrm{~dB}$. TC-SC-FDMA shows a $2 \mathrm{~dB}$ improvement in its variations, thanks to the use of pre-coding. As expected, TC-FSK and Turbo-FSK have the same performance, i.e. an IAPR of OdB. Both techniques rely on the use FSK modulation which exhibits a constant envelope.

The measure of the IAPR clearly emphasizes the main benefit of the proposed scheme, its constant amplitude property. It guarantees an IAPR equal to $0 \mathrm{~dB}$ for all samples. The theoretical efficiency of the PA can be shown conversely dependent on the level of IAPR [20]. Compared to TC-OFDM, the theoretical efficiency of the PA can be increased by $37 \%$ (resp. 56\%) for a class A (resp. class B) amplifier.

\section{CONCLUSION}

In the context of LPWA networks, reaching low levels of sensitivity and containing the power consumption at the device level is a major concern. Nonetheless, coexistence and integration into existing frameworks should be considered to facilitate the deployment procedure and reduce costs. In this work, we proposed the integration of Turbo-FSK in the exisiting 4G OFDM framework and demonstrated the possibility for the technique to use a transmitter and a receiver based on an OFDM-like architecture. Simulations demonstrated that the Turbo-FSK scheme achieves performance close to TC-OFDM in AWGN conditions and Rayleigh fading channels in both static and mobile conditions. However, while TC-OFDM exhibits important envelope variations, a known drawback for this modulation, the use of FSK provides a constant envelope and reduces the linearity constraints imposed on the PA. A theoretical efficiency of $90 \%$ can be achieved when considering a class B amplifier. Turbo-FSK trades a minimal loss in PER for a constant envelope, and is a very attractive alternative to classic OFDM. It is a promising additional mode for LPWA transmission that can be included in a scheduled network based on an OFDM framework. Turbo-FSK could be included in the 5G NB-IoT standard as a new low power mode significantly reducing the consumption at the device level, thanks to its constant envelope property. The proposed scheme allows to increase throughput and lower latency with respect to the single tone mode of NB-IoT while maintaining a constant envelope of the signal.

\section{ACKNOWLEDGEMENTS}

The research leading to these results received funding from the European Commission H2020 program under grant agreement number 723247 (5G-Champion project). This work was also supported by the French Agence Nationale de la Recheche (ANR), under grant agreement ANR-16-CE25-0002 (EPHYL project).

\section{REFERENCES}

[1] M. R. Palattella, M. Dohler, A. Grieco, G. Rizzo, J. Torsner, T. Engel and L. Ladid, "Internet of Things in the 5G Era: Enablers, Architecture, and Business Models," IEEE Journal on Selected Areas in Communications, vol. 34, no. 3, pp. 510-527, March 2016.

[2] International Telecommunication Union (ITU-T), "Overview of the Internet-of-Things," Recommendation ITU-T Y.2060, June 2012.

[3] U. Raza, P. Kulkarni, and M. Sooriyabandara, "Low Power Wide Area Networks: An Overview," IEEE Communications Surveys Tutorials, vol. PP, no. 99, pp. 1-1, 2017.

[4] H. P. Enterprise, "Low Power Wide Area (LPWA) Networks Play an Important Role in Connecting a Range of Devices," Business white paper, November 2016.

[5] T. Rebbeck, M. Mackenzie, and N. Afonso, "Low-Powered Wireless Solutions Have the Potential to Increase the M2M Market by Over 3 Billion Connections," Analysys Mason, Sept 2014.

[6] Y. Roth, J.-B. Doré, L. Ros, and V. Berg, "Turbo-FSK, a Physical Layer for Low-Power Wide-Area Networks: Analysis and Optimization," Elsevier Comptes Rendus Physique, vol. 18, no. 2, pp. 178 - 188, 2017, energy and radiosciences. [Online]. Available: http://www.sciencedirect.com/science/article/pii/S163107051630158X

[7] "LTE Evolved Universal Terrestrial Radio Access (E-UTRA): Physical Channels and Modulation," 3GPP TS 36.211, V13.2.0, Release 13, Aug 2016.

[8] "Whitepaper Narrowband Internet of Things," Rohde \& Schwarz, Aug 2016.

[9] R. Ratasuk, B. Vejlgaard, N. Mangalvedhe, and A. Ghosh, "NB-IoT System for M2M communication," in 2016 IEEE Wireless Communications and Networking Conference, April 2016, pp. 1-5.

[10] Y. P. E. Wang, X. Lin, A. Adhikary, A. Grovlen, Y. Sui, Y. Blankenship, J. Bergman, and H. S. Razaghi, "A Primer on 3GPP Narrowband Internet of Things," IEEE Communications Magazine, vol. 55, no. 3, pp. 117123, March 2017.

[11] C. Berrou, A. Glavieux, and P. Thitimajshima, "Near Shannon limit error-correcting coding and decoding: Turbo-codes," in IEEE International Conference on Communications (ICC). Geneva., vol. 2, May 1993, pp. 1064-1070.

[12] M. C. Valenti and J. Sun, "The UMTS turbo code and an efficient decoder implementation suitable for software defined radios," International Journal of Wireless Information Networks, vol. 8, pp. 203-216, 2001.

[13] Z. Wang and G. B. Giannakis, "Wireless multicarrier communications," IEEE Signal Processing Magazine, vol. 17, no. 3, pp. 29-48, May 2000.

[14] C. Shannon, "A mathematical theory of communication," The Bell System Technical Journal, vol. 27, no. 3, pp. 379-423, July 1948.

[15] Y. Roth, J.-B. Doré, L. Ros, and V. Berg, "Turbo-FSK: A New Uplink Scheme for Low Power Wide Area Networks," in 2015 IEEE 16th International Workshop on Signal Processing Advances in Wireless Communications (SPAWC), Stockholm, Sweden, June 2015, pp. 81-85.

[16] _ _ "EXIT Chart Optimization of Turbo-FSK: Application to Low Power Wide Area Networks," in 9th International Symposium on Turbo Codes \& Iterative Information Processing 2016 (ISTC'16), Brest, France, Sep. 2016

[17] "Evolved Universal Terrestrial Radio Access (E-UTRA): Base Station (BS) Radio Transmission and Reception," 3GPP TS 36.104, V14.3.0, Release 14, March 2017.

[18] J. Proakis, Digital Communications 3rd Edition, ser. Communications and signal processing. McGraw-Hill, 1995.

[19] P. Bento, J. Nunes, M. Gomes, R. Dinis, and V. Silva, "Measuring the magnitude of envelope fluctuations: Should we use the papr?" in 2014 IEEE 80th Vehicular Technology Conference (VTC2014-Fall), Sept 2014, pp. $1-5$

[20] S. L. Miller and R. J. O'Dea, "Peak power and bandwidth efficient linear modulation," IEEE Transactions on Communications, vol. 46, no. 12, pp. 1639-1648, Dec 1998. 